\title{
Effects of molecular markers on the treatment decision and prognosis of colorectal cancer: a narrative review
}

\author{
Yujuan Cao, Xiaodong Wang \\ Department of Oncology, Peking University Shougang Hospital, Beijing, China \\ Contributions: (I) Conception and design: Both authors; (II) Administrative support: X Wang; (III) Provision of study materials or patients: Y Cao; (IV) \\ Collection and assembly of data: Y Cao; (V) Data analysis and interpretation: Y Cao; (VI) Manuscript writing: Both authors; (VII) Final approval of \\ manuscript: Both authors. \\ Correspondence to: Xiaodong Wang. Department of Oncology, Peking University Shougang Hospital, No. 9 Jinyuanzhuang Road, Shijingshan \\ District, Beijing 100141, China. Email: xdwang2000@163.com.
}

\begin{abstract}
Objective: To summarize the effects of molecular markers on the treatment decision and prognosis of colorectal cancer.

Background: Colorectal cancer is a highly heterogeneous disease. Even colorectal cancers of the same pathological type and clinical stage may have significant differences in treatment efficacy and prognosis. There are three main molecular mechanisms for the occurrence and development of colorectal cancer: chromosomal instability (CIN) pathway, microsatellite instability (MSI), and CpG island methylate phenotype (CIMP). There are multiple molecular markers distributed on each pathway.

Methods: We performed a literature search on the PubMed database for studies published in English (from the date of initiation of the database to the year of 2020) using the following subject terms: "colon cancer", "rectal cancer", “colorectal cancer”, "molecular markers", "biomarkers", "treatment strategies”, and "prognosis".

Conclusions: The different expression states of molecular markers have a significant impact on the treatment decision and prognosis of colorectal cancer. Main colorectal cancer molecular markers include MSI and some important genes. Individualized treatments for tumors with different molecular phenotypes have improved the treatment effectiveness for colorectal cancer. The rational use of molecular markers is valuable for treatment decision-making and the prognosis of patients with colorectal cancer.
\end{abstract}

Keywords: Colorectal cancer; molecular markers; treatment strategy; prognosis

Submitted Mar 01, 2021. Accepted for publication May 26, 2021.

doi: 10.21037/jgo-21-230

View this article at: https://dx.doi.org/10.21037/jgo-21-230

\section{Introduction}

Colorectal cancer is one of the most common malignant tumors. It poses a serious threat to human health, ranking fourth among male malignancies, third among female malignancies, and has the fourth highest mortality of all malignant tumors $(1,2)$. Among first-time diagnosed patients with colorectal cancer, approximately twothirds are in stages II and III, and these patients have the opportunity to undergo radical surgical treatment. However, one-third of patients are at stage IV, and require comprehensive treatment. Colorectal cancer has a high degree of heterogeneity in terms of treatment effectiveness and prognosis. Even for colorectal cancers with the same pathological type and clinical stage, the curative effect and prognosis may be significantly different (3).

With the development of precision medicine, it has been discovered that the different states of colorectal cancer molecular markers have a significant impact on the treatment decision and prognosis this malignancy. Therefore, clarifying the expression of colorectal cancer molecular markers as soon 
as possible, predicting high-risk factors for recurrence and metastasis, and determining the sensitivity to treatment can help to guide individualized treatment of colorectal cancers with different molecular characteristics, and also predict the prognosis of the tumor to a certain extent. This article will specifically analyze the value and significance of microsatellite status, epidermal growth factor receptor $(E G F R)$, Kirsten ratsarcoma $(K R A S)$, v-raf murine sarcoma viral oncogene homolog B1 (BRAF), phosphatidylinositol-3-kinase catalytic (PIK3CA), phosphatase and tension (PTEN), and human epidermal growth factor receptor 2 (HER2) genes based on the important molecular markers in the clinical diagnosis and treatment of colorectal cancer. The focus of this review is on the treatment decision and prognosis prediction for patients with colorectal cancer. We present the following article in accordance with the Narrative Review reporting checklist (available at https://dx.doi.org/10.21037/jgo-21-230).

\section{Methods}

We performed a literature search on the PubMed database for studies published in English (from the date of inception of the database to 2020) using the following subject terms: "colon cancer", "rectal cancer", "colorectal cancer", "molecular markers", "biomarkers", "treatment strategies", and "prognosis".

\section{Discussion}

\section{The molecular mechanism of the occurrence and development of colorectal cancer}

Recent studies have identified three main molecular mechanisms for the occurrence and development of colorectal cancer: the chromosomal instability (CIN) pathway, microsatellite instability pathway (MSI), and CpG island methylate phenotype (CIMP) pathway $(1,3,4)$. These three pathways are not completely independent from each other or are solely present in one patient or one tumor, and some tumors have characteristics of multiple pathways.

\section{CIN pathway}

This is the most common pathway, accounting for approximately $70 \%$ of pathway involvement in colorectal cancer. It is characterized by chromosome rearrangement, loss of heterozygosity at tumor suppressor gene locus, and abnormal mutations of proto-oncogenes (such as $K R A S$, $B R A F$, PIK3CA) (4).

\section{MSI pathway}

Microsatellites are also called simple repetitive sequences. The insertion or deletion of simple repetitive sequences produced during DNA replication is called MSI, which is the accumulation of mutations caused by functional defects of the DNA mismatch repair (MMR) system. The incidence of MSI in colorectal cancer is approximately $15 \%(4,5)$. The 2020 China Colorectal Cancer Clinical Guidelines (6) recommend the use of the five detection sites proposed by the National Cancer Institute (NCI) (BAT25, BAT26, D5S346, D2S123, and D17S250). An evaluation of MSI status is made according to the instability of the site, and is characterized as one of the following three levels: microsatellite stability (MSS), low microsatellite instability (MSI-L), and high microsatellite instability (MSI-H).

The MSI status can also be reflected by detecting the absence of MMR proteins. The expression of four common MMR proteins [mutL homolog 1 (MLH1), mutS homolog 2 (MSH2), mutS homolog 6 (MSH6), and postmeiotic segregation increased 2 (PMS2)] can be detected by immunohistochemistry, and positive expression is located in the nucleus. The loss of expression of any protein is defective in mismatch repair function (dMMR), and the positive expression of all four proteins signifies intact mismatch repair function (pMMR). Generally speaking, $\mathrm{dMMR}$ is equivalent to MSI-H, and $\mathrm{pMMR}$ is equivalent to MSI-L or MSS.

\section{CIMP pathway}

CpG islands are widely methylated in DNA, and the incidence of CIMP in colorectal cancer is approximately $15-20 \%$ (4). This pathway is divided into CIMP-H and CIMP-L according to the amount of gene methylation (7-10). CIMP-H is usually associated with $B R A F$ mutations. Numerous sporadic MSIs are also CIMPs, which are usually located in the semicolon (8).

\section{The clinical significance of colorectal cancer molecular markers}

\section{MSI}

MSI is more common in the right colon, and in poorly differentiated pathological types, such as mucinous adenocarcinoma and medullary carcinoma, which are the characteristics of Lynch syndrome (11). Different stages of colorectal cancer have varying proportions of MSI, with the highest proportion in stage II patients (12). MSI can be used as a marker for whether patients with stage II 
colorectal cancer receive adjuvant chemotherapy. Studies have shown that MSI-H colorectal cancer is not sensitive to fluorouracil (FU)-based adjuvant chemotherapy and cannot benefit from FU adjuvant therapy (13). In patients with stage II colorectal cancer, MSI exhibits independent prognostic value. The overall survival (OS) and disease-free survival (DFS) of MSI patients are significantly better than those with MSS (13).

MSI can also be used as a predictor of the efficacy of programmed death 1 (PD-1) treatment in patients with advanced colorectal cancer. Programmed death receptor 1 is an important immunosuppressive molecule on the surface of T cells. Programmed cell death 1 ligand 1 (PD-L1) on the surface of tumor cells binds to PD- 1 on the surface of $\mathrm{T}$ cells to inhibit their growth. Immunity weakens the killing of tumor cells by $\mathrm{T}$ cells, and anti-PD-1 therapy can have an anti-tumor effect. The stable state of microsatellites is closely related to the effect of anti-PD-1 treatment. Studies have found that the effect of anti-PD-1 treatment in MSI patients is satisfactory. A study by scholars from Johns Hopkins University (14) found that for patients with MSItype advanced colorectal cancer who received anti-PD-1 treatment, the response rate was $40 \%$, and the progressionfree survival (PFS) rate at 20 weeks was $78 \%$.

\section{EGFR gene}

$E G F R$ is a transmembrane tyrosine kinase receptor, belonging to the epidermal growth factor receptor (ErbB) family of cell expression receptors [including EGFR (also called HER1 or ErbB-1), as well as HER2 (ErbB-2), HER3 (ErbB-3), HER4 (ErbB-4)]. When the ligand binds to EGFR, it activates its downstream pathway (15), which are mainly mitogen-activated protein kinases (MAPK) signal pathways and PI3K pathways. The activation of these pathways will in turn cause the activation of cell-related factors, resulting in cell proliferation, differentiation, metastasis, and apoptosis (16). Studies have found that EGFR is positively expressed in 25-35\% of colorectal cancers (17-19). Colorectal cancer patients with positive EGFR expression are poorly differentiated (8), and the prognosis of such patients is poor $(17,19)$. Clinical studies have found that positive $E G F R$ expression is not correlated with whether anti-EGFR targeted therapy is effective $(20,21)$.

\section{KRAS gene}

The $K R A S$ gene is a downstream gene of $E G F R$ and is a part of the MAPK signaling pathway. Approximately $30-40 \%$ of colorectal cancer patients have KRAS gene mutations (22).
The high incidence of mutations in the KRAS gene is located at codon $12 / 13$ in exon 2 . When the KRAS gene is mutated, GTPase activity is suppressed, and thus the KRAS protein is always activated, which causes abnormalities in the downstream signaling pathways, ultimately resulting in poor anti-EGFR treatment effectiveness $(23,24)$ or even worse $(25,26)$. A phase III clinical study found that in the postoperative adjuvant treatment of KRAS wildtype III colorectal cancer patients, the DFS was similar in patients treated with chemotherapy combined with antiEGFR adjuvant treatment compared to those who received chemotherapy alone (HR $=1.05 ; 95 \%$ CI: 0.85-1.29; $\mathrm{P}=0.66$ ) (27). In patients with advanced colorectal and KRAS mutations, first-line chemotherapy combined with antiEGFR therapy has prolonged PFS, compared with patients with chemotherapy alone (9.9 vs. 8.7 months, $\mathrm{P}=0.048$ ) (28). It is clear that anti-EGFR is only suitable for palliative chemotherapy for patients with advanced colorectal cancer. However, whether KRAS has a predictive effect on the prognosis of colorectal cancer remains controversial. Studies have found that patients with EGFR mutations have a shorter OS period $(29,30)$, while other studies do not support this conclusion $(31,32)$. Therefore, further research is needed to confirm these findings.

\section{$B R A F$ gene}

The RAF family is downstream of the $R A S$ gene, and there are three types: $A R A F, B R A F$, and $C R A F$ (33). BRAF is a part of the RAS-RAF-MAP2K (MEK)-MAPK signal pathway, which plays an important role in the occurrence and development of colorectal cancer. The most common BRAF mutation site is V600E, which also leads to the continuous activation of $R A S /$ RAF/MEK/ERK genes (33). BRAF V600E gene mutations are found in $5-10 \%$ of colorectal cancers, primarily in elderly female patients with proximal colon cancer. Furthermore, they are enriched in MSI patients, and most of them are mutually exclusive with $R A S$ gene mutations. Approximately 5-15\% of KRAS wild-type colorectal cancer patients have $B R A F$ mutations, and the anti-EGFR treatment effect for this type of patients is not good $(34,35)$.

BRAF gene mutation status has no guiding significance in the treatment of colorectal cancer, however it does have predictive significance for the prognosis of colorectal cancer. BRAF V600E mutation is a poor prognostic factor of early colorectal cancer and is related to the unstable state of microsatellites. The OS rate of MSS patients with $B R A F$ V600E mutation is significantly lower than that of nonmutated patients, while in MSI patients, there is no significant 
difference in OS compared to non-mutated patients $(31,36)$. For $K R A S$ wild-type advanced metastatic colorectal cancer patients, the OS and PFS of BRAF V600E mutation patients are lower than those of non-mutated patients (37).

\section{PIK3CA and PTEN gene}

The PI3K-AKT pathway is another EGFR signaling pathway, which can be regulated by PIK3CA mutation and PTEN gene inactivation $(15,38)$. Approximately $14-18 \%$ of colon cancer patients have PIK3CA mutations. Most PIK3CA mutations are exon 9 and 20 mutations $(35,39)$. Exon 9 mutations are often associated with KRAS mutations (35), and colon cancer patients with KRAS mutations and PIK3CA 9 exon mutations have poor survival rates (39). KRAS wild-type patients with exon 20 mutations have poor anti-EGFR treatment effects (35).

The PTEN protein, encoded by the PTEN gene, can inhibit the function of PI3K (15). When the PTEN gene is mutated, the inhibitory function disappears, leading to continuous activation of the PI3K-AKT pathway. The mutation rate of the PTEN gene in colorectal cancer is 13$18 \%(40,41)$. In $K R A S$ wild-type patients, the OS of patients with insufficient PTEN protein expression is lower than that of patients with normal PTEN protein expression (37).

\section{HER 2 gene}

The Human Cystic Echinococcosis ReseArch in CentraL and Eastern Societies (HERACLES) study found that in $K R A S$ wild-type advanced colorectal cancer patients, HER2 positive patients accounted for $5 \%$. These patients were not sensitive to anti-EGFR treatment, while cetuximab combined with apatinib treatment was clinically effective, with an objective response rate (ORR) of $35 \%$ (42). It is clear that the HER2 gene expression status has reference value for the diagnosis and treatment of colorectal cancer, although further research is needed.

In recent years, some new and effective non-invasive and highly compliant inspection methods such as plasma or stool test are gradually being used for colorectal cancer screening. Reagent test kit can test some CRC-related gene mutations or methylation (43). Studies also demonstrated that dynamic monitoring technology also has high application value in the prognostic judgment of colorectal cancer and the monitoring of recurrence and metastasis (44).

\section{Conclusions}

With the development of precision medicine, the diagnosis and treatment of malignant tumors tends toward molecularlevel research and individualized treatment. Therefore, clarifying the clinical guidance value and prognostic significance of molecular markers in colorectal cancer is crucial, so as to provide a basis for clinical decisionmaking, in order to maximize the survival time of patients and improve their quality of life. At present, it has been determined that the molecular markers mentioned in this study have a significant impact on the occurrence and development of colorectal cancer, as well as on the prognosis of diagnosis and treatment. However, experimental data and large-scale clinical trials are needed to verify the clinical significance of some molecular markers. Colorectal cancer is a heterogeneous disease, and its occurrence and development is affected by several factors. At present, this tumor has not been overcome, and thus, it remains necessary to continue to identify new targets to initiate the new chapter of tumor treatment.

\section{Acknowledgments}

Funding: Capital Clinical Characteristic Application Research and Achievement Promotion Project (No. Z16110000516003); Beijing Shijingshan District Medical Key Support Specialty Fund (No. 2016YFC1302600).

\section{Footnote}

Reporting Checklist: The authors have completed the Narrative Review reporting checklist. Available at https:// dx.doi.org/10.21037/jgo-21-230

Conflicts of Interest: Both authors have completed the ICMJE uniform disclosure form (available at https://dx.doi. org/10.21037/jgo-21-230). The authors have no conflicts of interest to declare.

Ethical Statement: The authors are accountable for all aspects of the work in ensuring that questions related to the accuracy or integrity of any part of the work are appropriately investigated and resolved.

Open Access Statement: This is an Open Access article distributed in accordance with the Creative Commons Attribution-NonCommercial-NoDerivs 4.0 International License (CC BY-NC-ND 4.0), which permits the noncommercial replication and distribution of the article with the strict proviso that no changes or edits are made and the 
original work is properly cited (including links to both the formal publication through the relevant DOI and the license). See: https://creativecommons.org/licenses/by-nc-nd/4.0/.

\section{References}

1. Boyle P, Langman JS. ABC of colorectal cancer: epidemiology. BMJ 2000;321:805-8.

2. Jemal A, Siegel R, Ward E, et al. Cancer statistics, 2008. CA Cancer J Clin 2008;58:71-96.

3. Bae JM, Kim JH, Kang GH. Molecular subtypes of colorectal cancer and their clinicopathologic features, with an emphasis on the serrated neoplasia pathway. Arch Pathol Lab Med 2016;140:406-12.

4. Mohammed AA, El-Tanni H, El-Khatib HM, et al. WITHDRAWN: Molecular classification of colorectal cancer: current perspectives and controversies. J Egypt Natl Canc Inst 2016. [Epub ahead of print]. doi: 10.1016/ j.jnci.2015.11.004.

5. Lengauer C, Kinzler KW, Vogelstein B. Genetic instability in colorectal cancers. Nature 1997;386:623-7.

6. National Health Commission of the People's Republic of China. Chinese Protocol of Diagnosis and Treatment of Colorectal Cancer (2020 edition). Zhonghua Wai Ke Za Zhi 2020;58:561-85.

7. Jass JR. Colorectal cancer: a multi pathway disease. Crit Rev Oncog 2006;12:273-87.

8. Wu C, Bekaii-Saab T. CpG island methylation, microsatellite instability, and BRAF mutations and their clinical application in the treatment of colon cancer. Chemother Res Pract 2012;2012:359041.

9. Shen L, Toyota M, Kondo Y, et al. Integrated genetic and epigenetic analysis identifies three different subclasses of colon cancer. Proc Natl Acad Sci USA 2007;104:18654-9.

10. Marzouk O, Schofield J. Review of histopathological and molecular prognostic features in colorectal cancer. Cancers 2011;3:2767-810.

11. Weisenberger DJ, Siegmund KD, Campan M, et al. $\mathrm{CpG}$ island methylator phenotype underlies sporadic microsatellite instability and is tightly associated with BRAF mutation in colorectal cancer. Nat Genet 2006;38:787-93.

12. Sinicrope FA, Foster NR, Thibodeau SN, et al. DNA mismatch repair status and colon cancer recurrence and survival in clinical trials of 5-fluorouracil-based adjuvant therapy. J Natl Cancer Inst 2011;103:863-75. Erratum in: J Natl Cancer Inst 2011;103:1639.

13. Klingbiel D, Saridaki Z, Roth AD, et al. Prognosis of stage
II and III colon cancer treated with adjuvant 5-fluorouracil or FOLFIRI in relation to microsatellite status: results of the PETACC-3 trial. Ann Oncol 2015;26:126-32.

14. Le DT, Uram JN, Wang H, et al. PD-1 blockade in tumors with mismatch-repair deficiency. N Engl J Med 2015;372:2509-20.

15. Hynes NE, Lane HA. ERBB receptors and cancer: the complexity of targeted inhibitors. Nat Rev Cancer 2005;5:341-54.

16. Citri A, Yarden Y. EGF-ERBB signalling: towards the systems level. Nat Rev Mol Cell Biol 2006;7:505-16.

17. Goldstein NS, Armin M. Epidermal growth factor receptor immunohistochemical reactivity in patients with American Joint Committee on Cancer Stage IV colon adenocarcinoma: implications for a standardized scoring system. Cancer 2001;92:1331-46.

18. McKay JA, Murray LJ, Curran S, et al. Evaluation of the epidermal growth factor receptor (EGFR) in colorectal tumors and lymph node metastases. Eur J Cancer 2002;38:2258-64.

19. Resnick MB, Routhier J, Konkin T, et al. Epidermal growth factor receptor, c-MET, -catenin, and p53 expression as prognostic indicators in stage II colon cancer: a tissue microarray study. Clin Cancer Res 2004;10:3069-75.

20. Chung KY, Shia J, Kemeny NE, et al. Cetuximab shows activity in colorectal cancer patients with tumors that do not express the epidermal growth factor receptor by immunohistochemistry. J Clin Oncol 2005;23:1803-10.

21. Cunningham D, Humblet Y, Siena S, et al. Cetuximab monotherapy and cetuximab plus irinotecan in irinotecanrefractory metastatic colorectal cancer. $\mathrm{N}$ Engl J Med 2004:351:337-45.

22. Burmer GC, Loeb LA. Mutations in the KRAS2 oncogene during progressive stages of human colon carcinoma. Proc Natl Acad Sci U S A 1989;86:2403-7.

23. Cappuzzo F, Finocchiaro G, Rossi E, et al. EGFR FISH assay predict for response to cetuximab in chemotherapy refractory colorectal cancer patients. Ann Oncol 2008;19:717-23.

24. Bardelli A, Siena S. Molecular mechanisms of resistance to cetuximab and panitumumab in colorectal cancer. J Clin Oncol 2010;28:1254-61.

25. Leslie A, Carey FA, Pratt NR, et al. The colorectal adenoma-carcinoma sequence. Br J Surg 2002;89:845-60.

26. Brink M, de Goeij AFPM, Weijenberg MP, et al. K-ras oncogene mutations in sporadic colorectal cancer in The Netherlands Cohort Study. Carcinogenesis 2003;24:703-10. 
27. Taieb J, Tabernero J, Mini E, et al. Oxaliplatin, fluorouracil, and leucovorin with or without cetuximab in patients with resected stage III colon cancer (PETACC-8): an open-label, randomised phase 3 trial. Lancet Oncol 2014;15:862-73.

28. Van Cutsem E, Köhne CH, Hitre E, et al. Cetuximab and chemotherapy as initial treatment for metastatic colorectal cancer. N Engl J Med 2009;360:1408-17.

29. Samowitz WS, Curtin K, Schaffer D, et al. Relationship of Ki-ras mutations in colon cancers to tumor location, stage, and survival: a population-based study. Cancer Epidemiol Biomarkers Prev 2000;9:1193-7.

30. Andreyev HJN, Norman AR, Cunningham D, et al. Kirsten ras mutations in patients with colorectal cancer: the 'RASCAL II' study. Br J Cancer 2001;85:692-6.

31. Roth AD, Tejpar S, Delorenzi M, et al. Prognostic role of KRAS and BRAF in stage II and III resected colon cancer: results of the translational study on the PETACC-3, EORTC 40993, SAKK 60-00 trial. J Clin Oncol 2010;28:466-74.

32. Tejpar S, Bertagnolli M, Bosman F, et al. Prognostic and predictive biomarkers in resected colon cancer: current status and future perspectives for integrating genomics into biomarker discovery. Oncologist 2010;15:390-404.

33. Holderfield M, Deuker MM, McCormick F, et al. Targeting RAF kinases for cancer therapy: BRAF-mutated melanoma and beyond. Nat Rev Cancer 2014;14:455-67.

34. Loupakis F, Ruzzo A, Cremolini C, et al. KRAS codon 61, 146 and BRAF mutations predict resistance to cetuximab plus irinotecan in KRAS codon 12 and 13 wild-type metastatic colorectal cancer. Br J Cancer 2009;101:715-21.

35. De Roock W, Claes B, Bernasconi D, et al. Effects of KRAS, BRAF, NRAS, and PIK3CA mutations on the efficacy of cetuximab plus chemotherapy in chemotherapyrefractory metastatic colorectal cancer: a retrospective consortium analysis. Lancet Oncol 2010;11:753-62.

Cite this article as: Cao Y, Wang X. Effects of molecular markers on the treatment decision and prognosis of colorectal cancer: a narrative review. J Gastrointest Oncol 2021;12(3):11911196. doi: 10.21037/jgo-21-230
36. Samowitz WS, Sweeney C, Herrick J, et al. Poor survival associated with the BRAF V600E mutation in microsatellite-stable colon cancers. Cancer Res 2005;65:6063-9.

37. Laurent-Puig P, Cayre A, Manceau G, et al. Analysis of PTEN, BRAF, and EGFR status in determining benefit from cetuximab therapy in wild-type KRAS metastatic colon cancer. J Clin Oncol 2009;27:5924-30.

38. Baba Y, Nosho K, Shima K, et al. Phosphorylated AKT expression is associated with PIK3CA mutation, low stage, and favorable outcome in 717 colorectal cancers. Cancer 2011;117:1399-408.

39. Ogino S, Nosho K, Kirkner GJ, et al. PIK3CA mutation is associated with poor prognosis among patients with curatively resected colon cancer. J Clin Oncol 2009;27:1477-84.

40. Zhou XP, Loukola A, Salovaara R, et al. PTEN mutational spectra, expression levels, and subcellular localization in microsatellite stable and unstable colorectal cancers. Am J Pathol 2002;161:439-47.

41. Nassif NT, Lobo GP, Wu X, et al. PTEN mutations are common in sporadic microsatellite stable colorectal cancer. Oncogene 2004;23:617-28.

42. Salvatore Siena AS, Sara Lonardi LT, Cosimo Martino $\mathrm{KB}$, et al. Trastuzumab and lapatinib in HER-2-amplified metastatic colorectal cancer patients (mCRC): the HERACLES trial. J Clin Oncol 2015;33 suppl: abstr 3508.

43. Imperiale TF, Ransohoff DF, Itzkowitz SH, et al. Multitarget stool DNA testing for colorectal-cancer screening. N Engl J Med 2014;370:1287-97.

44. Bettegowda C, Sausen M, Leary RJ, et al. Detection of circulating tumor DNA in early- and late-stage human malignancies. Sci Transl Med 2014;6:224ra24.

(English Language Editor: A. Kassem) 